Check for updates

Cite this: RSC Adv., 2017, 7, 42979

\title{
Synthesis of magnetic polymeric ionic liquid nanocomposites by the Radziszewski reaction $\uparrow$
}

\author{
Alexander Castro-Grijalba, ${ }^{a}$ Emilia M. Reyes-Gallardo, ${ }^{b}$ Rodolfo G. Wuilloud, (D) a \\ Rafael Lucena (D)*b and Soledad Cárdenas (D)*b
}

In the present article a new approach for the synthesis of magnetic polymeric ionic liquid nanocomposites based on the Radziszewski reaction is presented. The Radziszewski reaction is a simple and successful procedure to obtain imidazolium-containing polymers under mild conditions and consists of the reaction between diamine compounds, glyoxal and formaldehyde in an acidic media. If magnetic nanoparticles coated with amino groups are introduced, they can participate as polyamines in the synthesis thus providing a final nanocomposite where polymeric ionic liquids and nanoparticles are combined. The resulting nanocomposite has been characterized using several instrumental techniques aimed at elucidating its chemical composition (infrared spectroscopy and elemental analysis), properties (zeta potential) and morphology (microscopy, X-ray diffraction and dynamic light scattering). Finally, its sorptive ability towards three non-steroidal anti-inflammatory drugs (NSAIDs) such as salicylic acid, ketoprofen and naproxen was evaluated considering the main variables involved in the process. According to the results, the polymeric ionic liquid nanocomposite is a promising sorptive material for analytical purposes.

Received 19th July 2017

Accepted 31st August 2017

DOI: $10.1039 / \mathrm{c} 7 \mathrm{ra0} 0794 \mathrm{~h}$

rsc.li/rsc-advances with an enhanced mechanical stability. ${ }^{13}$ PILs are usually synthesized by radical polymerization using monomers containing acryloyl, methacryloyl or vinyl groups. These procedures, which are time consuming and needs a lot of resources (reagents, solvents, high temperatures), provide low chemical yields and require several purification stages. ${ }^{14}$ However, J. P. Lindner ${ }^{15}$ has recently proposed the synthesis of imidazoliumbased polymers via the Radziszewski reaction. The reaction that can be developed at room temperature in $24 \mathrm{~h}$ can be also accelerated if the temperature is increased at $100{ }^{\circ} \mathrm{C}$. The reaction yields (as high as 98\%), the simplicity of the reaction conditions and the low reagents requirements make this synthetic approach highly attractive.

Polymeric nanocomposites, comprising polymers and nanoparticles, have recently attracted much attention in the sample preparation context thanks to their special properties ${ }^{\mathbf{1 6}}$ such as the mechanical, ${ }^{17}$ thermal, ${ }^{18}$ photoluminescence ${ }^{19}$ and thermoelectric ones. ${ }^{\mathbf{2 0}}$ The polymeric part usually offers the main interaction chemistry ${ }^{21}$ while the nanometric domain improves this performance (adding a secondary interaction or enhancing the superficial areas) $)^{22,23}$ or provides the nanocomposite with special properties (e.g. magnetism). ${ }^{24}$ In fact, although PILs can be directly used in sample preparation, ${ }^{25,26}$ their combination with nanoparticles drives to special and improved materials. ${ }^{27}$ Magnetic polymeric nanocomposites are especially attractive in sample treatment since they allow the simplification of the sample processing. In this sense, the nanocomposite can be easily dispersed into the sample
Facultad de Ciencias Exactas y Naturales, Universidad Nacional de Cuyo, Instituto Interdisciplinario de Ciencias Básicas (ICB), UNCUYO-CONICET, Padre J. Contreras 1300, (5500) Mendoza, Argentina

${ }^{b}$ Departamento de Quimica Analitica, Instituto Universitario de Investigación en Química Fina y Nanoquímica IUIQFN, Universidad de Córdoba, Campus de Rabanales, Edificio Marie Curie, E-14071 Córdoba, Spain. E-mail: rafael.lucena@ uco.es; scardenas@uco.es

$\dagger$ Electronic supplementary information (ESI) available. See DOI: 10.1039/c7ra07947h 
enlarging the analyte/sorbent contact while it can be easily recovered using an external magnetic field. ${ }^{28}$ Core-shell approach, consisting of coating the surface of the magnetic nanoparticle (MNP) with an appropriate phase, is the most common approach. Although it has several advantages, the functionalization of the MNPs with PILs is not an easy task. For example, Zheng et al. $^{29}$ employed vinyltriethoxysilane as a previous step to attach the $\mathrm{PIL}$ to $\mathrm{Fe}_{3} \mathrm{O}_{4} @ \mathrm{SiO}_{2}$ requiring as
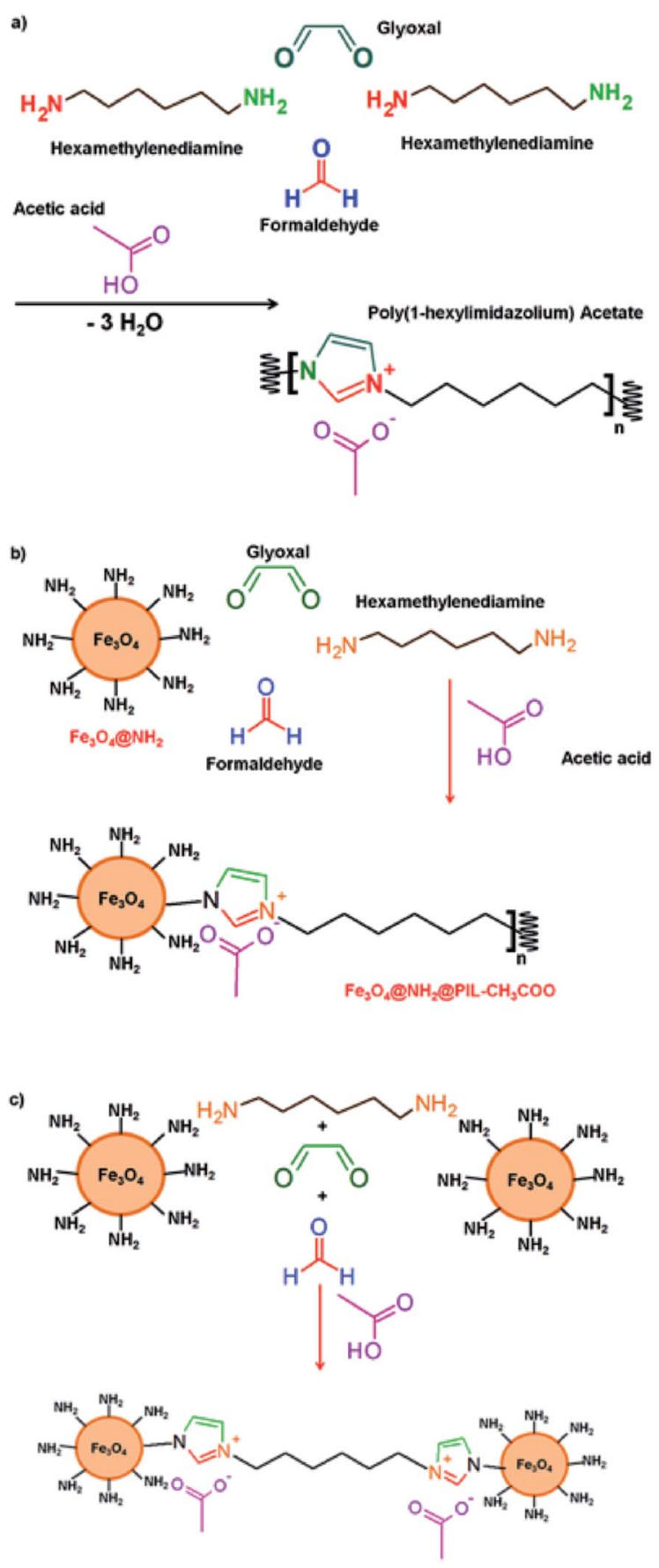

Fig. 1 Schematic diagram of the synthesis of polymeric ionic liquids (PILs) by the Radziszewski reaction. Panel (a) describes the synthesis of bulk PILS while panels ( $b$ and $c$ ) show potential paths for the synthesis of the nanocomposites. catalyst triethanolamine in distilled toluene under inert atmosphere at $100{ }^{\circ} \mathrm{C}$ and a reaction time of $24 \mathrm{~h}$. Then, the PIL was attached to this structure by a reaction at $100{ }^{\circ} \mathrm{C}$ for $2 \mathrm{~h}$ under nitrogen atmosphere. The PIL was formed by free radical copolymerization of the monomer unit of 1-vinyl-3hexylimidazolium bromide employing 2,2-azobisisobutyronitrile as initiator. The complete process took $42 \mathrm{~h}$, without considering the time for washing and purification steps. Other authors $s^{27,30}$ have reported similar reaction times, temperatures and inert atmosphere to obtain the final PIL magnetic nanocomposite.

In this work, a new PIL magnetic nanocomposite was obtained based in the Radziszewski reaction. ${ }^{15}$ This approach simplifies the overall synthetic procedure reducing the amount of reagents and the synthesis time. The bulk synthesis involves the reaction between diamine compounds, glyoxal and formaldehyde in an acidic media as it is shown in Fig. 1a. Our approach is based on the use of magnetic nanoparticles $\left(\mathrm{Fe}_{3} \mathrm{O}_{4}\right)$ coated with amine groups that may be involved as polyamines in the Radziszewski reaction following some synthetic paths as those described in Fig. 1b and c. The resulting material has been characterized by different instrumental techniques and its potential use as sorbent towards three nonsteroidal antiinflammatory drugs (NSAIDs) (salicylic acid, ketoprofen and naproxen) in saliva samples has been outlined.

\section{Experimental section}

\section{Reagents and samples}

All reagents were of analytical grade or better. Unless otherwise specified, they were purchased from Sigma-Aldrich (Madrid, Spain). Stock standard solution of each analyte (salicylic acid, ketoprofen and naproxen) was prepared at a concentration of $1000 \mathrm{mg} \mathrm{L}^{-1}$ in methanol (Carlo Erba Reagents, Italy) and stored at $4{ }^{\circ} \mathrm{C}$. A diluted solution containing all the analytes at a concentration of $100 \mathrm{mg} \mathrm{L}^{-1}$ was prepared in methanol and stored at $4{ }^{\circ} \mathrm{C}$. Working solutions were prepared by the appropriate dilution of the stocks in Milli-Q water (Millipore Corp., Madrid, Spain). Acetonitrile, acetic acid (both from Panreac, Barcelona, Spain), and ultrapure water were employed to prepare the chromatographic mobile phase. Iron(III) chloride hexahydrate $\left(\mathrm{FeCl}_{3} \cdot 6 \mathrm{H}_{2} \mathrm{O}\right)(99 \%)$, iron(II) chloride tetrahydrate $\left(\mathrm{FeCl}_{2} \cdot 4 \mathrm{H}_{2} \mathrm{O}\right)(99.99 \%)$ and ammonia $(25 \%)$ were used for the synthesis of the magnetic nanoparticles $\left(\mathrm{Fe}_{3} \mathrm{O}_{4}\right)$. (3-Aminopropyl)triethoxysilane (APTES) (98\%) and ethanol were employed for coating the nanoparticles with a protective silicabased coating. For the synthesis of PIL, the following reagents were used: glyoxal (40\%), acetic acid (99\%), formaldehyde (37\%) and hexamethylenediamine $(98 \%)$.

Blank saliva samples were collected from healthy volunteers, using a citric acid solution as stimulus. Food and liquids (apart from water) ingestion, chewing gum and tooth brushing were avoided $30 \mathrm{~min}$ before sampling. The whole saliva $(2.5-3 \mathrm{~mL}$ approximately) was obtained by spitting into a polypropylene commercial sterile pot (Deltalab, Barcelona, Spain) and stored at $4{ }^{\circ} \mathrm{C}$ until the final analysis (performed in the same day of 
collection). The samples were filtered through $0.22 \mu \mathrm{m}$ regenerated cellulose filters.

\section{Synthesis of the magnetic polymeric ionic liquid nanocomposite}

Synthesis of magnetic nanoparticles. The synthesis of MNPs was carried out by a co-precipitation method. A total of $24 \mathrm{~g}$ of $\mathrm{FeCl}_{3} \cdot 6 \mathrm{H}_{2} \mathrm{O}$ and $9.8 \mathrm{~g}$ of $\mathrm{FeCl}_{2} \cdot 4 \mathrm{H}_{2} \mathrm{O}$ were dissolved in $100 \mathrm{~mL}$ of Milli-Q water. The mixture was maintained during $30 \mathrm{~min}$ at $80{ }^{\circ} \mathrm{C}$ under nitrogen atmosphere. Then, $50 \mathrm{~mL}$ of ammonia (25 $\mathrm{wt} \%)$ was added dropwise with a syringe producing a black precipitate of iron oxide. The MNPs formed were separated by an external magnet $(60 \times 30 \times 15 \mathrm{~mm}$ and $549.4 \mathrm{~N}$ of maximum adhesive force), washed with water (three-times) and acetonitrile and finally dried at $80^{\circ} \mathrm{C}$ for $24 \mathrm{~h}$.

In order to protect them, the MNPs were coated with a silica shell with amino $\left(-\mathrm{NH}_{2}\right)$ groups. $4 \mathrm{~g}$ of the MNPs was added to an ethanol/water (200 and $16 \mathrm{~mL}$, respectively) solution. This solution was sonicated for $30 \mathrm{~min}$ to ensure the total dispersion of the MNPs. Then, $8 \mathrm{~mL}$ of 3-aminopropyl-triethoxysilane (APTES) was added. After that, the solution was stirred during $24 \mathrm{~h}$ under nitrogen atmosphere. The final material $\mathrm{Fe}_{3} \mathrm{O}_{4} @ \mathrm{NH}_{2}$, was recovered with an external magnet and washed with water (at least six-times) and acetonitrile (twice). Finally, the solid was dried in an oven at $80^{\circ} \mathrm{C}$ for $24 \mathrm{~h}$.

In situ synthesis of the nanocomposite. For the synthesis of magnetic polymeric ionic liquid nanocomposite (MPILNC), $500 \mathrm{mg}$ of $\mathrm{Fe}_{3} \mathrm{O}_{4} @ \mathrm{NH}_{2}$ was added in a solution of $1.46 \mathrm{~g}$ of water, $978 \mathrm{mg}$ of hexamethylenediamine and $101 \mathrm{mg}$ of acetic acid. Hexamethylenediamine was melted first at $60{ }^{\circ} \mathrm{C}$. This solution was sonicated for $1 \mathrm{~h}$. Then, a mixture of $1.22 \mathrm{~g}$ of glyoxal solution (40\%) with $682 \mathrm{mg}$ of formaldehyde $(37 \%)$ was added. After that, the final mixture was sonicated for $6 \mathrm{~h}$ and then, stirred for $18 \mathrm{~h}$ at $25^{\circ} \mathrm{C}$. The final MPILNC, was recovered with an external magnet and washed with water (at least sixtimes) and acetonitrile (twice). Finally, the solid was dried in an oven at $80{ }^{\circ} \mathrm{C}$ for $24 \mathrm{~h}$.

\section{Characterization of the magnetic polymeric ionic liquid nanocomposite}

The MPILNC was characterized using several instrumental techniques aimed at knowing its chemical composition (infrared spectroscopy and elemental analysis), properties (zeta potential) and morphology (microscopy, X-ray diffraction and dynamic light scattering).

Infrared measurements were performed in a Bruker Tensor 37 FT-IR spectrometer, equipped with a diamond ATR cell with a circular surface of $3 \mathrm{~mm}$ diameter and three internal reflections. A Deuterated Triglycine Sulfate (DTGS) detector was employed for spectra acquisition. Spectra were collected between 4000 and $600 \mathrm{~cm}^{-1}$ at a $4 \mathrm{~cm}^{-1}$ resolution with 64 coadded scans each. Data collection was made using OPUS software (Bruker, Ettligen, Germany). Elemental analysis was carried out on an elemental analyzer CHNS Eurovector EA 3000, which was used for measuring the carbon and nitrogen content of the synthesized nanomaterials.
A Philips CM-10 transmission electron microscopy (TEM) was employed to characterize their particle size, morphology and aggregation behavior of MPILNC. X-ray powder diffraction (XRD) patterns were recorded on a Siemens D5000 X-ray diffractometer, using $\mathrm{Cu} \mathrm{K} \alpha$ radiation and a graphite monochromator. The average hydrodynamic diameter, size distribution an zeta potential of the nanocomposites in aqueous samples were calculated at room temperature, using a Malvern Zetasizer Nano ZSP ZEN5600 analyzer equipped with a $10 \mathrm{~mW}$ laser He-Ne $(633 \mathrm{~nm})$. A Vibracell ${ }^{\mathrm{TM}} 75.041$ ultrasonic probe ( $750 \mathrm{~W}, 20 \mathrm{KHz}$, Bioblock Scientific, Illkirch, France) equipped with a $3 \mathrm{~mm}$ probe was employed for the dispersion of the suspensions.

The micrographs acquisition and elemental analysis measurements were developed in the Central Service for Research Support (SCAI) of the University of Córdoba. Zetapotential, X-ray diffraction and dynamic light scattering measurements were done at the Institute of Fine Chemistry and Nanochemistry of the University of Cordoba.

\section{Chromatographic analysis}

Liquid chromatographic analysis was performed using a HP1100 series liquid chromatograph (Agilent, Palo Alto, CA) equipped with a binary high-pressure pump for mobile phase delivery, an autosampler and a single wavelength photometer (HP1100 series). Data analysis was performed using HP ChemStation software. Chromatographic separation was performed with a LiChrosorb C18 $(4.6 \mathrm{~mm} \times 250 \mathrm{~mm}, 5 \mu \mathrm{m}, 100 \AA)$ column (Analisis Vinicos, Tomelloso, Spain), using acetonitrile and a $3 \%$ acetic acid aqueous solution as mobile phase components in a $42 / 58(\mathrm{v} / \mathrm{v})$ ratio. The injection volume was $10 \mu \mathrm{L}$, the flow rate was $1.2 \mathrm{~mL} \mathrm{~min}^{-1}$ in isocratic mode and the analytes were monitored at $254 \mathrm{~nm}$.

\section{Results and discussion}

\section{Synthesis and characterization of magnetic polymeric ionic liquid nanocomposite}

Radziszewski reaction is an easy and efficient procedure to synthesize imidazolium-containing polymers under mild conditions. The reaction between diamine compounds, glyoxal and formaldehyde in an acidic media drives to the formation of a PIL as it is shown in Fig. 1a. The acid used in the synthesis defines the counteranion of the resulting PIL. This synthesis has been practically evaluated in the lab to demonstrate the feasibility and the synthesis procedure as well as the IR characterization of the PIL are included in the ESI (Section S1 and Fig. S1). $\dagger$

The hypothesis tested in this article consists of using magnetic nanoparticles coated with amine groups as reagent in this synthesis with the aim to obtain a polymeric nanocomposite where nanoparticles and PIL chains are combined. Theoretically, the amino groups, one or several of them, located over the surface of the NP can act as reagents of the Radziszewski reaction to form the imidazolium rings with another amino group from the diamine of $\mathrm{C} 6$ chain and initiate the 
polymerization onto the surface of the nanoparticles as it is shown in Fig. 1b. In addition, the PIL chain presents a terminal amino group that can interact with another hexamethylenediamine molecule or even with another nanoparticle as it is indicated in Fig. 1c. This strategy showed an easier route for the synthesis of these nanomaterials than the explored before by other authors. ${ }^{29,31}$

Fig. 2 compares the IR spectra for the initial nanoparticles and the resulting nanocomposite. The characteristic peaks for $\mathrm{Fe}_{3} \mathrm{O}_{4} @ \mathrm{NH}_{2}$ (Fig. 2a) appeared at 585-632 $\mathrm{cm}^{-1}$ for the stretching vibrations of Fe-O-Fe, at $1000-1200 \mathrm{~cm}^{-1}$ for the $\mathrm{Si}-\mathrm{O}, \mathrm{Si}-\mathrm{O}-\mathrm{H}$ vibrations, at $1625 \mathrm{~cm}^{-1}$ for the bending mode of $\mathrm{NH}_{2}$ and at $3400 \mathrm{~cm}^{-1}$ region for the stretching vibration of $\mathrm{N}-\mathrm{H}$. All these peaks showed the efficient coating of the MNPs with APTES. The IR spectrum of the nanocomposite (Fig. 2b) indicated two new peaks with medium absorbance at 1382 and $1558 \mathrm{~cm}^{-1}$ corresponding to the stretching vibrations of $\mathrm{C}-\mathrm{N}$ of the imidazolium ring and the $\mathrm{C}=\mathrm{O}$ of the acetate group, respectively. These results confirmed the functionalization of the surface of MNPs with PIL. Other characteristic peaks of $\mathrm{Fe}_{3} \mathrm{O}_{4} @ \mathrm{NH}_{2}$ nanoparticles were present in the region of $585-632 \mathrm{~cm}^{-1}$ (Fe-O bonds) and 1000-1200 $\mathrm{cm}^{-1}$ (Si-O, Si-O-H

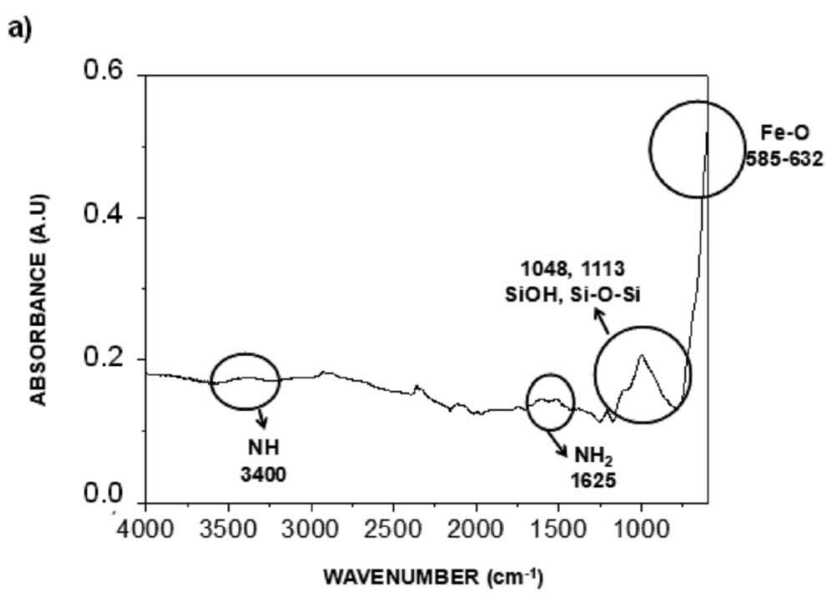

b)

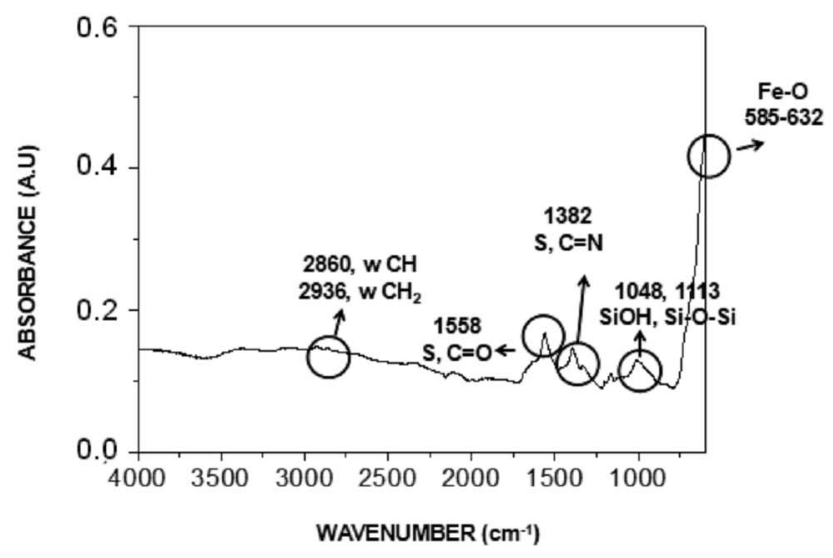

Fig. 2 ATR-FTIR spectra of (a) initial nanoparticles and (b) nanocomposite. The main IR absorption bands for each material are identified. bonds). The small bands at 2860 and $2936 \mathrm{~cm}^{-1}$ correspond to the asymmetric and symmetric stretching modes of $-\mathrm{CH}_{2}-$ vibrations. ${ }^{32}$

The XRD spectra of initial nanoparticles and the nanocomposite are shown in Fig. S2. $\dagger$ The six characteristic peaks appear at $2 \theta$ of $30.1^{\circ}, 35.6^{\circ}, 43.4^{\circ}, 54.0^{\circ}, 57.0^{\circ}$ and $62.7^{\circ}$. The nanocomposite and the initial nanoparticles present similar diffraction peaks, indicating that the crystalline structure of the nanoparticles does not change after functionalization with the PIL. The average magnetic particle size $(L)$ can be estimated using Scherrer's equation relating the coherently scattering domains with Bragg peak widths: $L=k \lambda / B \cos (\theta)$, where $k$ is Scherrer constant, $\lambda$ the X-ray wavelength, $\beta$ the peak width of half maximum and $\theta$ is the Bragg diffraction angle. The average magnetic particle size obtained from this equation was $11.9 \mathrm{~nm}$.

The size and surface morphology of the initial NPs and the nanocomposite were evaluated by transmission electron microscopy (TEM). Fig. 3a shows that the $\mathrm{Fe}_{3} \mathrm{O}_{4} @ \mathrm{NH}_{2}$ nanoparticles were spherical, dispersed and with an average diameter of $10 \mathrm{~nm}$. This size is in accordance with the obtained value by Scherrer's equation with the results by XRD. Only few agglomerates were observed. On the other hand, Fig. 3b shows that nanoparticles were strongly agglomerated in the nanocomposite indicating, as it was expected in the initial hypothesis, that the PIL acted as an entanglement agent between the
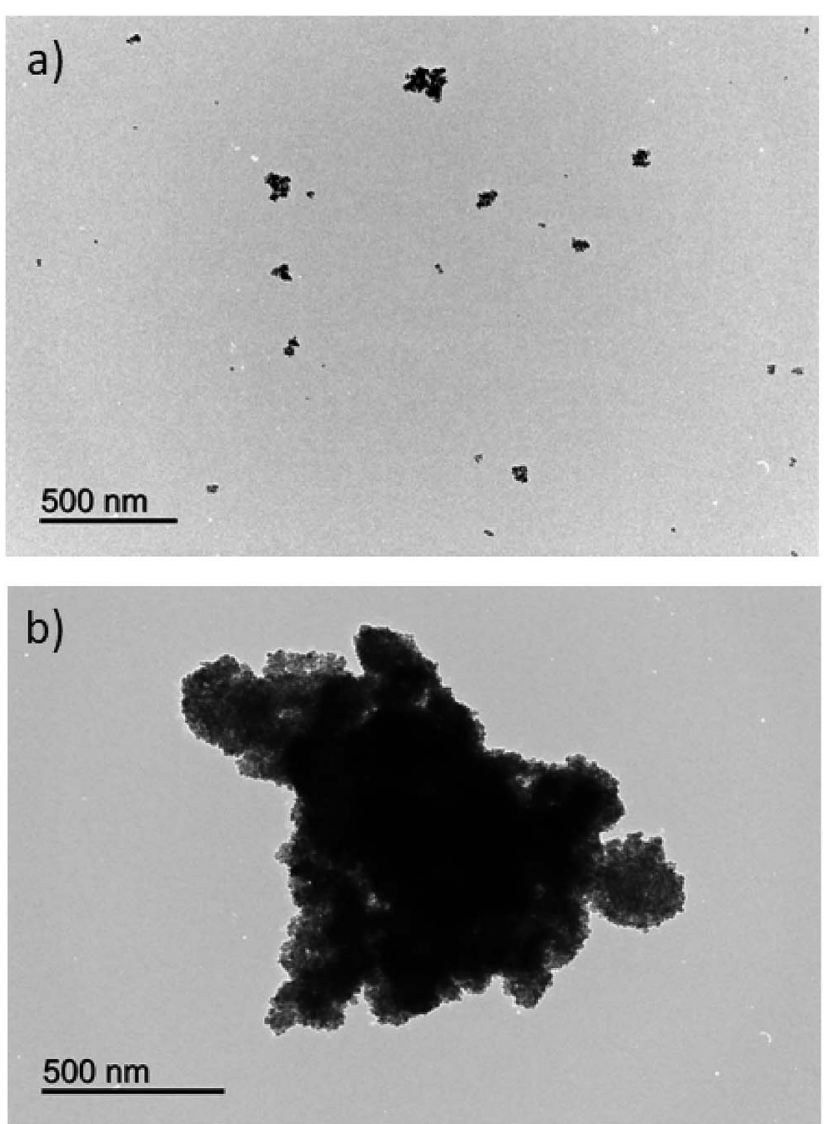

Fig. 3 TEM micrographs of the (a) initial nanoparticles and (b) nanocomposite. 
$\mathrm{Fe}_{3} \mathrm{O}_{4} @ \mathrm{NH}_{2}$ nanoparticles. The size of magnetic nanocomposite was larger than the $\mathrm{Fe}_{3} \mathrm{O}_{4} @ \mathrm{NH}_{2}$ nanoparticles and resulted between 500 and $1000 \mathrm{~nm}$.

These observations were confirmed by dynamic light scattering (DLS) measurement as it is shown in Fig. 4. The hydrodynamic radius $\left(R_{\mathrm{H}}\right)$, which is the radius of the hypothetical hard sphere that diffuses with the same speed as the particles assayed under DLS, ${ }^{33}$ varies between both type of materials. In this sense, the $R_{\mathrm{H}}$ of the nanoparticles was $137.7 \mathrm{~nm}$ while the nanocomposite presents a $R_{\mathrm{H}}$ of $883.4 \mathrm{~nm}$. In addition the broader peaks obtained for the nanocomposite indicates a lower homogeneity than the original nanoparticles. This effect was expected since the synthesis of the nanocomposite combines two different paths (presented in Fig. 1b and c). As it was expected, the hydrodynamic ratio by DLS was larger than the size observed by TEM. This size is larger than observed by TEM due to the hydration ratio since the nanoparticles are charged with amino groups which have high affinity for water molecules increasing its hydration.

The $\mathrm{N} \%$ and $\mathrm{C} \%$ content can be used for characterization as well. The $\% \mathrm{~N}$ and \% $\mathrm{C}$ content of the initial nanoparticles, obtained by elemental analysis (see Experimental section), resulted to be $0.96 \%$ and $4.40 \%$ due to the presence of the

a)

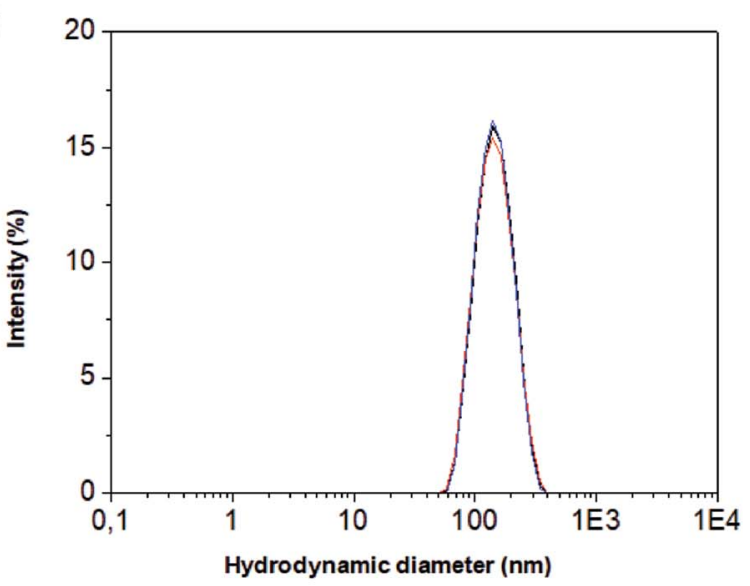

b)

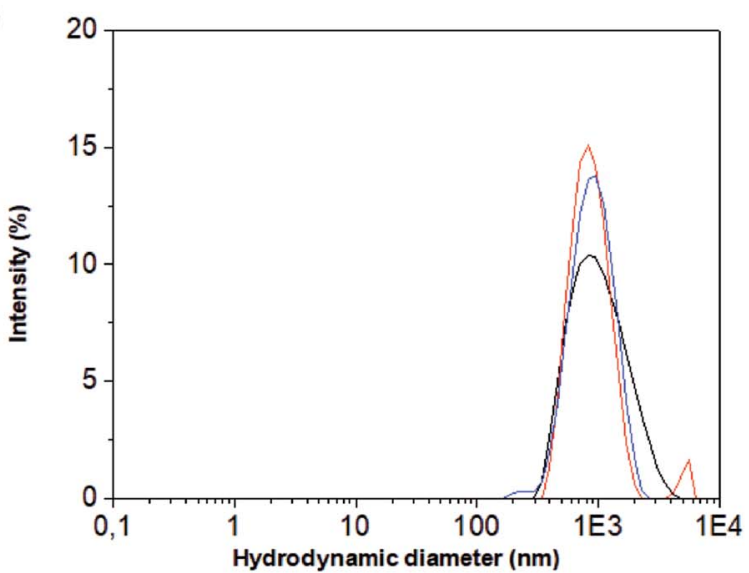

Fig. 4 Particle size distribution by DLS of the (a) initial nanoparticles and (b) nanocomposite.
APTES reagent. The percentages increased up to $1.42 \% \mathrm{~N}$ and $7.81 \% \mathrm{C}$ in the nanocomposite. This increment confirms once again the presence of the PIL in the nanocomposite. However, the increase (1.5 and 1.8 times, respectively) is not dramatic and it is line with the observation of bulk PIL in the supernatant of the synthesis.

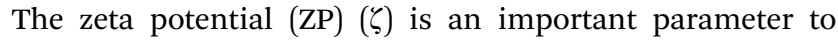
elucidate the stability of a dispersion of the nanoparticles in a specific medium. The $\mathrm{ZP}$ was evaluated at different $\mathrm{pHs}$ for the starting and final material, the results being shown in Fig. 5. The two materials show values higher than zero which indicate that both present a positive charge. The higher value obtained for the MPILNC can be ascribed to the imidazolium rings.

\section{Elucidation of the sorptive ability of the magnetic polymeric ionic liquid nanocomposite}

Once the material was characterized demonstrating its successful synthesis, its sorptive ability was evaluated. Three acidic compounds with moderate and $\mathrm{pH}$ dependent polarity were selected as model targets. They present a negative charge, complementary to the positive charge of the PIL, when the working $\mathrm{pH}$ is higher to their $\mathrm{p} K_{\mathrm{a}}$ values. Therefore they are interesting analytes to understand the potential interactions (reverse phase and/or ionic exchange mechanisms) with the sorptive phase. The analyte/nanocomposite interaction may be affected by several variables like those summarized in Table S1 (ESI $\dagger$ ). Table $\mathrm{S} 1 \dagger$ also presents the studied interval and the best results for each one. For this evaluation, an aqueous standard solution containing a mixture of all analytes at a concentration of $2 \mathrm{mg} \mathrm{L}^{-1}$ was employed. Separation and quantification of the analytes was performed by HPLC-UV-VIS. For brevity, only the key parameters will be discussed in the text.

$\mathrm{pH}$ is an important parameter because it establishes the anionic or neutral form of the analytes in solution, which have carboxylic groups in their structures. Salicylic acid has a $\mathrm{p} K_{\mathrm{a}}$ of 2.97 , ketoprofen of 3.80 and naproxen of $4.6 .^{34-36}$ The $\mathrm{pH}$ range evaluated was 2.0-8.0. The results of this evaluation are shown in Fig. 6a. The analyte extraction was almost constant for $\mathrm{pHs}$

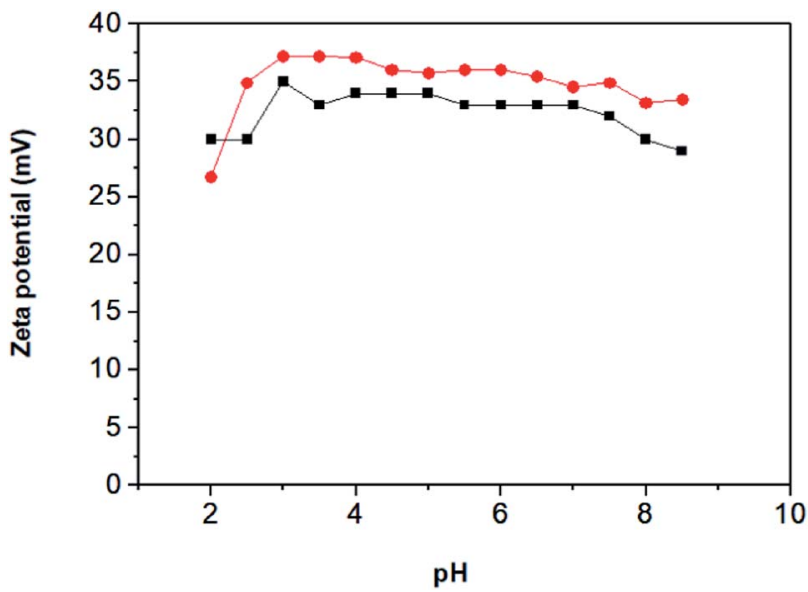

Fig. $5 \mathrm{Z}$ potential vs. pH for nanoparticles (๑). 

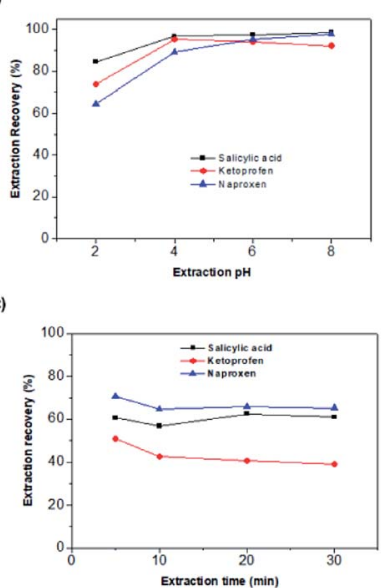

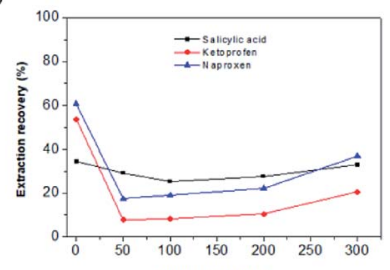

NaCl amount (g L-1)

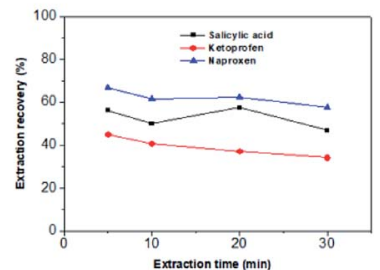

Fig. 6 (a) Effect of the pH on the extraction efficiency. (b) Influence of the ionic strength on the extraction of the analytes. (c) Effect of time on the extraction when vortex is used for agitation. (d) Effect of time on the extraction when ultrasounds are used for agitation.

higher than 6. The extraction was better at alkaline pHs indicating that the analytes must be in the anionic form to be extracted by the MPILNC, which is charged positively according to zeta potential results.

Ionic strength is a key parameter since the stability of the system is greatly influenced by the charged ions present in the media. This parameter also influences the affinity of the analytes for the MPILNC. The ionic strength was evaluated in the range 0 and $300 \mathrm{~g} \mathrm{~L}^{-1}$ using sodium chloride as model electrolyte. The results of the evaluation are shown in Fig. $6 \mathrm{~b}$ indicating a clear effect of the ionic strength on the extraction of the analytes.

The elution was performed by dispersing MPILNC after the extraction process in an appropriate solvent employing 1 min of ultrasound. The following mixtures were evaluated for this purpose: methanol/acetic acid $80: 20$, methanol, methanol/ ammonia $80: 20$ and acetonitrile. The highest elution recovery was obtained with the mixture methanol/acetic acid $80: 20$ with values of $35 \%, 54 \%$ and $62 \%$ for salicylic acid, ketoprofen and naproxen, respectively. The elution volume was studied in a range of $100-500 \mu \mathrm{L}$, being $500 \mu \mathrm{L}$ selected as the best one since a quantitative elution of the analytes was obtained with this volume. On the other hand, other methanol/ acetic acid ratios were evaluated but no further improvements were achieved.

The amount of MPILNC was evaluated in the range from 5 to $30 \mathrm{mg}$. The analytical signal for naproxen and ketoprofen do not vary with the amount of the nanocomposite indicating its good extraction capability. However, the background signal increases at short retention times with the amount of nanoparticles affecting the determination of salicylic acid. Therefore $5 \mathrm{mg}$ was selected as the best value.

Once the amount of MPILNC was studied, the type of agitation (vortex or ultrasound) and extraction time (5-30 $\mathrm{min}$ ) were evaluated. The results, which are shown in Fig. 6c and d, demonstrated that shorter times using vortex as agitation

provided the best results. Ultrasounds or longer time provided lower recoveries probably due to the slight heating of the solution under these conditions. These results show that the extraction could be achieved in a short time $(5 \mathrm{~min})$ indicating a good superficial area of the nanocomposite and the homogeneous dispersion of MPILNC in the solution during extraction. ${ }^{37}$

The sorptive ability of the MPILNC has been demonstrated studying as well the key variables involved. If this sorbent is intended to be used for analytical purposes, its sorptive capacity must answer to the concentration of the target analytes in the original sample. The extraction workflow presented in ESI $\dagger$ (Section 4) includes the optimum conditions of the above studied variables. It was applied to the extraction of aqueous standards and saliva samples (where these analytes may be found as a consequence of their therapeutic use) providing some interesting data. Saliva samples showed a strong matrix effect, compared with aqueous standards, that can be ascribed to their inherent viscosity. Viscous samples hinder the normal diffusion of the analytes to the sorptive phase reducing the extraction kinetics and therefore the efficiency. However, this problem can be overcome if a matrix-match calibration approach is used. For this purpose, blank saliva samples were spiked with the analytes in the range from 100 to $20000 \mu \mathrm{g} \mathrm{L}{ }^{-1}$. The calibration graphs were linear for all the analytes in all the range of evaluated concentration providing correlation coefficients in the range of 0.9968 and 0.9999 . These results are promising for the analytical application of the new nanocomposite.

\section{Conclusions}

In the present article, a nanocomposite comprising polymeric ionic liquids and magnetic nanoparticles is synthesized in a simple and rapid way. The synthesis is based on the use of magnetic nanoparticles coated with amine groups as polyamines in the Radziszewski reaction. The new material presented the synergic properties of nanoparticles (magnetic behavior) and polymeric ionic liquids (high affinity for the anionic analytes). The new material was characterized employing different techniques and its extraction performance was evaluated using three model analytes (salicylic acid, ketoprofen and naproxen). The high extraction results were achieved thanks to the positive charged surface of the nanocomposite in all the range of $\mathrm{pH}$ evaluated and to the stability of the nanoparticles. The nanocomposite showed its potential application to bioanalytical samples such as saliva. The short times of microextraction and the small amount of nanocomposite to be used in each single extraction can be highlighted as the main advantages of the analytical procedure.

\section{Conflicts of interest}

There are no conflicts to declare. 


\section{Acknowledgements}

Financial support from the Spanish Ministry of Economy, Industry and Competitiveness (grant CTQ2014-52939R) is gratefully acknowledged. E. M. Reyes Gallardo expresses her gratitude for the predoctoral grant (refs FPU12/02670) from the Spanish Ministry of Education. A. Castro-Grijalba expresses her gratitude for the teacher's mobility scholarship from Universidad Nacional de Cuyo and Patagonia Bank and for the doctoral scholarship from Consejo Nacional de Investigaciones Científicas y Técnicas (CONICET). The authors would like to thank the Central Service for Research Support (SCAI) and the Institute of Fine Chemistry and Nanochemistry, both at the University of Cordoba, for the services provided to characterize the nanocomposites.

\section{Notes and references}

1 M. Palacio and B. Bhushan, Adv. Mater., 2008, 20, 1194-1198. 2 T. D. Ho, A. J. Canestraro and J. L. Anderson, Anal. Chim. Acta, 2011, 695, 18-43.

3 N. V. Plechkova and K. R. Seddon, Chem. Soc. Rev., 2008, 37, 123-150.

4 E. Aguilera Herrador, R. Lucena, S. Cárdenas and M. Valcárcel, Trends Anal. Chem., 2010, 29, 602-616.

5 P. Berton, E. M. Martinis, L. D. Martinez and R. G. Wuilloud, Anal. Chim. Acta, 2009, 640, 4046.

6 C. F. Poole and S. K. Poole, J. Sep. Sci., 2011, 34, 888-900.

7 A. M. Stalcup and B. Cabovska, J. Liq. Chromatogr. Relat. Technol., 2004, 27, 1443-1459.

8 S. Hayashi and H. O. Hamaguchi, Chem. Lett., 2004, 33, 1590-1591.

9 R. E. Del Sesto, T. M. McCleskey, A. K. Burrell, G. A. Baker, J. D. Thompson, B. L. Scott, J. S. Wilkes and P. Williams, Chem. Commun., 2008, 4, 447-449.

10 K. D. Clark, O. Nacham, H. Yu, T. Li, M. M. Yamsek, D. R. Ronning and J. L. Anderson, Anal. Chem., 2015, 87, 1552-1559.

11 A. Chisvert, J. L. Benedé, J. L. Anderson, S. A. Pierson and A. Salvador, Anal. Chim. Acta, 2017, 983, 130-140.

12 R. Marcilla, J. A. Blazquez, J. Rodriguez, J. A. Pomposo and D. Mecerreyes, J. Polym. Sci., Part A: Polym. Chem., 2004, 42, 208-212.

13 D. Mecerreyes, Prog. Polym. Sci., 2011, 36, 1629-1648.

14 O. Green, S. Grubjesic, S. Lee and M. A. Firestone, Polym. Rev., 2009, 49, 339-360.
15 J. P. Lindner, Macromolecules, 2016, 49, 2046-2053.

16 E. Reyes-Gallardo, R. Lucena, S. Cárdenas and M. Valcárcel, Bioanalysis, 2015, 7, 1723-1730.

17 Z. Hu and G. Chen, Adv. Mater., 2014, 26, 5950-5956.

18 J. Liu, G. Chen and J. Yang, Polymer, 2008, 49, 3923-3927.

19 L. Wu, G. Chen and Z. Li, Small, 2017, 13, 1604070.

20 G. Chen, W. Xu and D. Zhu, J. Mater. Chem. C, 2017, 5, 43504360.

21 C. E. Nika, E. Yiantzi and E. Psillakis, Anal. Chim. Acta, 2016, 922, 30-36.

22 H. Bagheri, S. Banihashemi and F. K. Zandian, Microchim. Acta, 2016, 183, 195-202.

23 B. Fresco-Cala, S. Cardenas and J. M. Herrero-Martínez, Microchim. Acta, 2017, 184, 1863-1871.

24 E. Reyes-Gallardo, G. Lasarte-Aragonés, R. Lucena, S. Cárdenas and M. Valcárcel, J. Chromatogr. A, 2013, 1271, 50-55.

25 J. López-Darias, V. Pino, J. L. Anderson, C. M. Graham and A. M. Afonso, J. Chromatogr. A, 2010, 1217, 1236-1243.

26 Y. Meng and J. L. Anderson, J. Chromatogr. A, 2010, 1217, 6143-6152.

27 Y. Chen, S. Cao, L. Zhang, C. Xi, X. Li, Z. Chen and G. Wang, J. Chromatogr. A, 2016, 1448, 9-19.

28 E. Reyes-Gallardo, R. Lucena, S. Cárdenas and M. Valcárcel, J. Chromatogr. A, 2014, 1345, 43-49.

29 X. Zheng, L. He, Y. Duan, X. Jiang, G. Xiang, W. Zhao and S. Zhang, J. Chromatogr. A, 2014, 1358, 39-45.

30 X. Guo, H. Wu, S. Guo, Y. Shi, J. Du, P. Zhu and L. Du, Anal. Sci., 2016, 32, 763-768.

31 A. A. Asgharinezhad and H. Ebrahimzadeh, J. Chromatogr. A, 2016, 1435, 18-29.

32 C. Yuan, J. Zhang, G. Chen and J. Yang, Chem. Commun., 2011, 47, 899-901.

33 S. Bhattacharjee, J. Controlled Release, 2016, 235, 337-351.

34 H. Sevelius, R. Runkel, E. Segre and S. Bloomfield, Br. J. Clin. Pharmacol., 1980, 10, 259-263.

35 B. Savun-Hekimoğlu and N. H. Ince, Ultrason. Sonochem., 2017, 39, 243-249.

36 M. Lorier, L. Magallanes, M. Ibarra, N. Guevara, M. Vázquez and P. Fagiolino, Eur. J. Drug Metab. Pharmacokinet., 2016, 41, 787-793.

37 H. Parham and N. Rahbar, J. Pharm. Biomed. Anal., 2009, 50, 58-63. 